\title{
Epidemiología del suicidio en adolescentes y jóvenes en Ecuador
}

\author{
Rebekka Maria F. Gerstner, ${ }^{1}$ Ismael Soriano, ${ }^{2}$ Antonio Sanhueza, ${ }^{3}$ \\ Sonia Caffe ${ }^{3}$ y Devora Keste/ ${ }^{3}$
}

Forma de citar

Gerstner RMF, Soriano I, Sanhueza A, Caffe S, Kestel D. Epidemiología del suicidio de adolescentes y jóvenes en Ecuador. Rev Pan Am Salud Publica. 2018;42:e100. https:/ / doi.org/10.26633/RPSP.2018.100

RESUMEN Objetivo. Recabar y ofrecer información a quienes toman decisiones en los programas de salud en general, y de salud mental en particular, sobre las características epidemiológicas del suicidio de jóvenes y adolescentes ecuatorianos.

Método. Se realizó un estudio ecológico transversal con datos de los registros de mortalidad por suicidio de los jóvenes y adolescentes de Ecuador de 2001 a 2014. El análisis se desagregó por región natural, sexo, edad, grupo étnico y por el método usado para cometer el suicidio. Se estimaron tasas de mortalidad por suicidio (por 100000 habitantes) y los riesgos relativos $(R R)$ de suicidio por sexo y regiones.

Resultados. Entre 2001 y 2014 se registraron 4855 suicidios en adolescentes y jóvenes. El riesgo más alto se estimó en los varones de 15 a 24 años y en los adolescentes residentes en la Amazonía, seguidos por los de la Sierra. La etnia con más casos de suicidio fueron los mestizos, aunque $40 \%$ de los jóvenes que se suicidan en la Amazonía son indígenas. El método más frecuente fue el ahorcamiento seguido por el envenenamiento con pesticidas.

Conclusiones. El suicidio de adolescentes y jóvenes es un problema de salud pública importante en Ecuador. Como en él influyen diversos factores psicológicos, sociales y culturales, se observan grandes variaciones según la región, los grupos de edad y las etnias. La restricción del acceso a pesticidas y a otros productos químicos, los programas de prevención universales y en centros educativos en las áreas con las tasas más altas, y las intervenciones específicas dirigidas a grupos vulnerables podrían ayudar a reducir los suicidios de jóvenes en Ecuador.

Palabras clave Suicidio; adolescente; epidemiología; Ecuador.

El suicidio puede definirse como el resultado de comportamientos autoinfligidos que tienen como intención la propia muerte (1). Cada año mueren alrededor

\footnotetext{
Pontificia Universidad Católica del Ecuador, Sede Santo Domingo, Santo Domingo, Ecuador. La correspondencia se debe dirigir a Rebekka Maria F Gerstner remgerstner@gmail.com

2 Nutrition and Family, Gender \& Life Course, Pan American Health Organization, Quito, Ecuador.

3 Pan American Health Organization, Ringgold Standard Institution, Washington, DC, Estados Unidos de América.
}

de 800000 personas en el mundo por esta causa, pese a que se considera prevenible (2). El grupo de población de 15 a 24 años de edad ha recibido considerable atención de las autoridades sanitarias con respecto al suicidio, porque en él el suicidio es la segunda causa de muerte y porque, además, se ha registrado un aumento importante de su frecuencia en las últimas décadas, sobre todo en países de ingresos medios y bajos de Asia y América (2).
Algunos análisis sobre el suicidio de adolescentes y jóvenes indican que en ciertos países se notifican las tasas más altas a escala mundial $(1,2)$, como en Ecuador, donde en las últimas dos décadas casi 300 jóvenes y adolescentes de 10 a 24 años se quitaron la vida anualmente (3). Según el último informe sobre el suicidio en la Región de las Américas de la Organización Panamericana de la Salud (OPS) (4), las tasas de suicidio de adolescentes de 10 a 19 años

Este es un artículo de acceso abierto distribuido bajo los términos de la licencia Creative Commons Attribution-NonCommercial-NoDerivs 3.0 IGO, que permite su uso, distribución y reproducción en cualquier medio, siempre que el trabajo original se cite de la manera adecuada. No se permiten modificaciones a los artículos ni su uso comercial. Al reproducir un artículo no debe haber ningún indicio de que la OPS o el artículo avalan a una organización o un producto específico. El uso del logo de la OPS no está permitido. Esta leyenda debe conservarse, junto con la URL original del artículo. 
y de 15 a 24 de Ecuador fueron, respectivamente, 7,5 y 13,6 por 100 000. En sus países vecinos dichas tasas fueron 4,7 y 9,4 en Colombia y 1,1 y 1,9 en Perú, respectivamente. Además, según el mismo informe, el suicidio en Ecuador representa entre 15 y $20 \%$ de las muertes por causas externas en este grupo de edad, lo que también contrasta con Colombia, donde este porcentaje representa entre 7 y $11 \%$ de estos fallecimientos, y con Perú, donde oscila entre 6 y $7 \%$.

Algunas características culturales y geográficas podrían ser responsables de estas cifras elevadas. Ecuador es un país pluricultural: $7 \%$ de su población es afroamericana, $7 \%$, montubia, $6 \%$, blanca, $72 \%$, mestiza y $7 \%$, indígena. Las cuatro zonas geográficas en que se divide el país (Costa, Sierra, Amazonía y Región Insular) se caracterizan por tener un clima, la altitud, la flora y la fauna muy distintos. En un estudio realizado con la población general se han estimado tasas de suicidio más altas en la Sierra y en la Amazonía (la mayor parte de cuya población es indígena) que en la Costa y la Región Insular. En las dos primeras zonas predominan problemas sociales como la migración y el abuso de drogas y de alcohol, que, a su vez, están asociados con un elevado riesgo de suicidio (5).

El suicidio de jóvenes de diversos grupos étnicos, sobre todo indígenas, se considera un problema importante en muchos países occidentales, como, por ejemplo, en los Estados Unidos de América, Canadá, Australia y Nueva Zelanda, donde las tasas de suicidio en la población indígena son hasta cinco veces más altas que en el resto de la población (6). Lo mismo ocurre en Brasil, Colombia, Perú y Chile, en los cuales dichas tasas también son más altas en sus pueblos indígenas (7).

Respecto al suicidio de jóvenes y adolescentes en Ecuador, hasta la fecha se carece de información epidemiológica de alcance nacional que permita concentrar esfuerzos en las áreas que requieren mayor atención. Con el presente estudio se pretende recabar y ofrecer información a quienes toman decisiones en los programas de salud en general, y de salud mental en particular, sobre las características epidemiológicas del suicidio de jóvenes y adolescentes ecuatorianos entre 10 y 24 años de edad. Con esta información, se intentará, asimismo, resituar al suicidio en adolescentes y jóvenes como una prioridad de salud a la cual deben dirigirse mayores esfuerzos para la detección temprana del riesgo.

\section{Materiales y Métodos}

Se realizó un estudio ecológico transversal utilizando datos de los registros de mortalidad por suicidio de jóvenes y adolescentes de 2001 a 2014 del Instituto Nacional de Estadística y Censos de Ecuador. Se selecionaron las defunciones clasificadas con los códigos de causa básica de muerte de la CIE-10 (X60 - X84), teniendo en cuenta el total de las 4855 muertes por suicidio en las edades mencionadas registradas en dicho período. Las variables incluidas en el estudio fueron: edad, sexo, zona (urbana/rural), distribución geográfica (provincias y regiones), etnia, y causas de suicidio. Se consideró la mortalidad a partir de 2001 para evitar los problemas que pueden haberse producido con los datos como consecuencia del cambio de la CIE-9 a la CIE-10 en 1998.

La edad se definió como variable categórica (10-19 años, 10-24 años y 15-24 años), porque la Organización Mundial de la Salud (OMS) define como adolescentes a los que tienen de 10 a 19 años de edad y como jóvenes, a los que tienen entre 15 y 24 años. Estos grupos no son mutuamente excluyentes. Para describir el suicidio de todos los adolescentes y jóvenes se utilizó el grupo de 10 a 24 años, y para describirlo en los adolescentes y en los jóvenes por separado, los grupos de la OMS, de 10 a 19 y de 15 a 24 años, respectivamente. La variable distribución geográfica consta de tres niveles (Sierra, Amazonía y Costa) y en ella no se incluyó la región insular porque sólo tiene una provincia con una tasa de suicidios muy baja.

El análisis exploratorio de los datos consistió en calcular las tasas de mortalidad por suicidio (por 100000 habitantes) y medidas de tendencia central y de dispersión entre los años 2001 y 2014. Además, para estimar el riesgo relativo de suicidio por sexo y regiones se construyeron modelos de regresión de Poisson utilizando los datos entre los años 2008 y 2014. La bondad de ajuste de los modelos se evaluó con los criterios de información de Akaike, Akaike corregido y bayesiano. Todos los datos se analizaron utilizando los programas estadísticos SAS ${ }^{\circledR}$ y SPSS, versión 22.

\section{Resultados}

En Ecuador, entre 2001 y 2014 se registraron 4855 muertes por suicidio de adolescentes y jóvenes, 2738 de adolescentes (10-19 años) y 4144 de jóvenes (15-24 años). La mayoría de los fallecidos eran varones de 15 a 24 años de edad. La tasa de suicidio más alta fue 10,5 por 100000 en 2007 (13,4 en hombres y 7,7 en mujeres), seguida por 9,6 en 2012 (12,5 en hombres y 6,7 en mujeres), y la más baja, 6,4 (7,4 en hombres y 5,5 en mujeres), seguida por 6,5 en 2014 (8,6 en hombres y 4,4 en mujeres). El mayor porcentaje de suicidios se registró en zonas urbanas y el método más frecuente fue el ahorcamiento, seguido por el envenenamiento con pesticidas.

La tasa de mortalidad por suicidio de los jóvenes y adolescentes en Ecuador ha variado entre 2001 y 2014, con picos entre 2006 y 2008 y entre 2011 y 2012. Sin embargo, a partir de 2013, se aprecia una disminución del número de muertes por esta causa en jóvenes y adolescentes (de 10 a 24 años de edad).

Las tasas de mortalidad por suicidio por provincias cambiaron entre los períodos 2001-2007 y 2008-2014 (cuadro 1). En general se observa que en la Costa la tasa sufrió una leve disminución, mientras que en la Sierra aumentó $15 \%$ y en la Amazonía, 40\% (cuadro 1).

El riesgo de muerte por suicidio fue mayor en los hombres, pero varió según la edad (cuadro 2): entre los 10 y los 19 años de edad, los riesgos de los hombres eran algo mayores que los de las mujeres (1,3 veces más alto), mientras que entre los 15 y los 24 años el riesgo llegó a ser 1,9 veces más alto. Sin embargo, los IC $95 \%$ en estos dos grupos de edades se solapan, indicando que puede no haber diferencias de los riesgos entre estos grupos.

El riesgo de suicidio fue mayor en la Amazonía y en la Sierra que en la Costa (cuadro 2) y las diferencias entre las tres regiones fueron más pronunciadas en las personas de edades más tempranas y se redujeron en las de más edad. En cada grupo de edad no existen diferencias significativas de los riesgos en las regiones; esto debido a que los IC95\% de los RR en las regiones se solapan. La calibración de los modelos construidos es adecuada.

Respecto al método de suicidio, las diferencias por sexo eran notables: en las mujeres, el envenenamiento fue el método más frecuente $(54 \%)$, seguido por el ahorcamiento (41\%), y en los hombres el 
CUADRO 1. Tasas de mortalidad por suicidio por 100000 habitantes y variaciones en adolescentes y jóvenes de 10 a 24 años por provincia y período, Ecuador

\begin{tabular}{|c|c|c|c|}
\hline \multirow{2}{*}{ Provincias } & \multicolumn{2}{|c|}{ Período } & \multirow{2}{*}{$\begin{array}{c}\text { Cambio } \\
(\%)\end{array}$} \\
\hline & 2001-2007 & 2008-2014 & \\
\hline Azuay & 16,02 & 17,75 & 10,8 \\
\hline Bolívar & 7,06 & 16,11 & 128,2 \\
\hline Cañar & 14,63 & 18,1 & 23,7 \\
\hline Carchi & 16,59 & 21,8 & 31,4 \\
\hline Cotopaxi & 12,43 & 15,43 & 24,1 \\
\hline Chimborazo & 10,92 & 13,08 & 19,8 \\
\hline El Oro & 4,62 & 6,22 & 34,6 \\
\hline Esmeraldas & 10,37 & 6,50 & $-37,3$ \\
\hline Guayas & 6,23 & 4,65 & $-25,4$ \\
\hline Imbabura & 14,95 & 13,24 & $-11,4$ \\
\hline Loja & 7,46 & 7,14 & $-4,3$ \\
\hline Los Ríos & 7,29 & 6,67 & $-8,5$ \\
\hline Manabí & 5,00 & 4,09 & $-18,2$ \\
\hline Morona Santiago & 9,43 & 13,84 & 46,8 \\
\hline Napo & 12,73 & 18,24 & 43,3 \\
\hline Pastaza & 4,97 & 9,18 & 84,7 \\
\hline Pichincha & 10,79 & 8,12 & $-24,7$ \\
\hline Tungurahua & 13,84 & 13,01 & $-6,0$ \\
\hline Zamora & 6,15 & 9,09 & 35,0 \\
\hline Galápagos & 4,45 & 2,10 & 60,5 \\
\hline Sucumbíos & 13,71 & 12,79 & $-6,7$ \\
\hline Orellana & 11,06 & 18,27 & 65,2 \\
\hline Santo Domingo ${ }^{1}$ & & 8,72 & \\
\hline Santa Elena ${ }^{1}$ & & 0 & \\
\hline Media general & 10,03 & 11,48 & 14,5 \\
\hline Costa & 6,70 & 6,14 & $-8,4$ \\
\hline Sierra & 12,47 & 14,38 & 15,3 \\
\hline Amazonía & 9,68 & 13,57 & 40,2 \\
\hline
\end{tabular}

Fuente: Instituto Nacional de Estadística y Censos.

'Santo Domingo y Santa Elena se crearon como provincia en 2007.

CUADRO 2. Riesgos relativos de suicidio por grupo de edad, sexo y región de 2008 a 2014, Ecuador

\begin{tabular}{llcc}
\hline Grupo de edad (años) & \multicolumn{1}{c}{ Sexo } & Riesgo relativo & IC95\% \\
\hline 10 a 19 & Hombre & 1,3 & $(1,1-1,4)$ \\
& Mujer (referencia) & & \\
10 a 24 & Hombre & 1,8 & $(1,6-1,9)$ \\
& Mujer (referencia) & & \\
15 a 24 & Hombre & 1,9 & $(1,7-2,1)$ \\
& Mujer (referencia) & & $(2,8-3,5)$ \\
10 a 19 & Sierra & 3,1 & $(3,0-4,4)$ \\
& Amazonia & 3,7 & $(2,3-2,8)$ \\
10 a 24 & Costa (referencia) & & $(2,6-3,5)$ \\
& Sierra & 2,5 & $(2,2-2,7)$ \\
15 a 24 & Amazonia & 3,0 & $(2,4-3,3)$ \\
& Costa (referencia) & & \\
\hline
\end{tabular}

Fuente: Instituto Nacional de Estadística y Censos.

más frecuente fueron los ahorcamientos $(66 \%)$, seguido por el envenenamiento con pesticidas $(25 \%)$ y los disparos (5\%).
Entre las zonas urbanas y rurales no existieron diferencias importantes en cuanto a los métodos utilizados, salvo un porcentaje un poco más elevado de envenenamientos en la zona rural $(40 \%)$ que en la urbana (34\%). En esta última, los ahorcamientos fueron más frecuentes que en la rural (59y $53 \%$, respectivamente) respecto al total de suicidios. La distribución porcentual de los demás métodos de suicidio fue similar en ambas zonas (cuadro 3). En la Amazonía y en la Sierra prevaleció el envenenamiento como método más frecuente (figura 2), aunque en las provincias con mayor concentración de zonas urbanas (Guayas, Pichincha y Azuay) el envenenamiento (con pesticidas, medicamentos y otras sustancias químicas) solo alcanzó entre 20 y 30\% de todos los casos.

En relación con la etnia, se observaron grandes diferencias entre las tres regiones naturales. En todas ellas, la etnia con mayor porcentaje de suicidio fueron los mestizos (48\% en la Amazonía, 74\% en la Costa, y $75 \%$ en la Sierra), seguida por los indígenas (50\% en la Amazonia y $15 \%$ en la Sierra). En la Costa, entre 2010 y 2014, 12\% de las muertes por suicidio se produjeron en la población afroecuatoriana.

\section{Discusión}

Entre los dos períodos (2001-2007 y 2008-2014), el suicidio de jóvenes y adolescentes en Ecuador aumentó de 11 a $27 \%$ según el grupo considerado. Las tasas de mortalidad por suicidio variaron notablemente por provincias y por grupos de edad, pero la tendencia general muestra un aumento de dichas tasas en los jóvenes y adolescentes ecuatorianos.

La razón de sexos fue menor que la notificada en otros países de la Región, como Argentina, Venezuela, Uruguay y Colombia, donde oscilan en torno a 1,8 (en el grupo de 10 a 19 años de edad) y a 3,9 (en el de 20 a 24), y se acerca a las de Perú, El Salvador, Paraguay (4) y a las publicadas en algunos países de Asia, como Corea y Japón, donde se encuentran entre 1,0 y 2,7 en los grupos de edad mencionados (8).

Hasta la década de los noventa, Ecuador ha sido uno de los pocos países del mundo donde las tasas de suicidio de las mujeres jóvenes y adolescentes eran más altas que en los hombres de estos mismos grupos de edad (9). A partir de 2006, con los cambios políticos y socioeconómicos que se produjeron en el país, se redujeron las tasas de suicidio de las mujeres y aumentaron las de los hombres (3) 
CUADRO 3. Métodos de suicidio por sexo y zona en la población de 10 a 24 años de edad entre 2008 y 2014, Ecuador

\begin{tabular}{|c|c|c|c|c|c|c|c|c|}
\hline \multirow{2}{*}{ Método de suicidio (Código CIE-10) } & \multicolumn{2}{|c|}{ Hombres } & \multicolumn{2}{|c|}{ Mujeres } & \multicolumn{2}{|c|}{ Urbano } & \multicolumn{2}{|c|}{ Rural } \\
\hline & No. & $\%$ & No. & $\%$ & No. & $\%$ & No. & $\%$ \\
\hline X60-64 Envenenamientos medicamentos & 13 & 0,8 & 17 & 1,9 & 22 & 1,3 & 7 & $\overline{0,9}$ \\
\hline X65-69 Envenenamientos pesticidas & 405 & 25,4 & 486 & 54,5 & 586 & 33,9 & 296 & 39,4 \\
\hline X70 Ahorcamientos & 1052 & 65,9 & 369 & 41,4 & 1021 & 59,0 & 400 & 53,2 \\
\hline X71 Ahogamientos & 1 & 0,1 & 0 & 0 & 1 & 0,1 & 0 & 0 \\
\hline X72-74 Disparos & 77 & 4,8 & 6 & 0,7 & 65 & 3,8 & 26 & 3,5 \\
\hline X75-77 Exposición a fuego, humo o explosivos & 11 & 0,7 & 0 & 0,0 & 1 & 0,1 & 0 & 0,0 \\
\hline X78-79 Objetos cortantes o romos & 5 & 0,3 & 3 & 0,3 & 5 & 0,3 & 1 & 0,1 \\
\hline X80-82 Caídas, saltos, colisiones & 5 & 0,3 & 0 & 0,0 & 5 & 0,3 & 1 & 0,1 \\
\hline X83-84 Eventos no especificados & 27 & 1,7 & 11 & 1,2 & 24 & 1,4 & 21 & 2,8 \\
\hline Total & 1596 & 100 & 892 & 100 & 1730 & 100 & 752 & 100 \\
\hline
\end{tabular}

Fuente: Instituto Nacional de Estadística y Censos.

FIGURA 1. Tasas de mortalidad por suicidio por 100000 habitantes en Ecuador por región y provincia para las edades entre 10 y 24 años (medias del período 2008-2014)

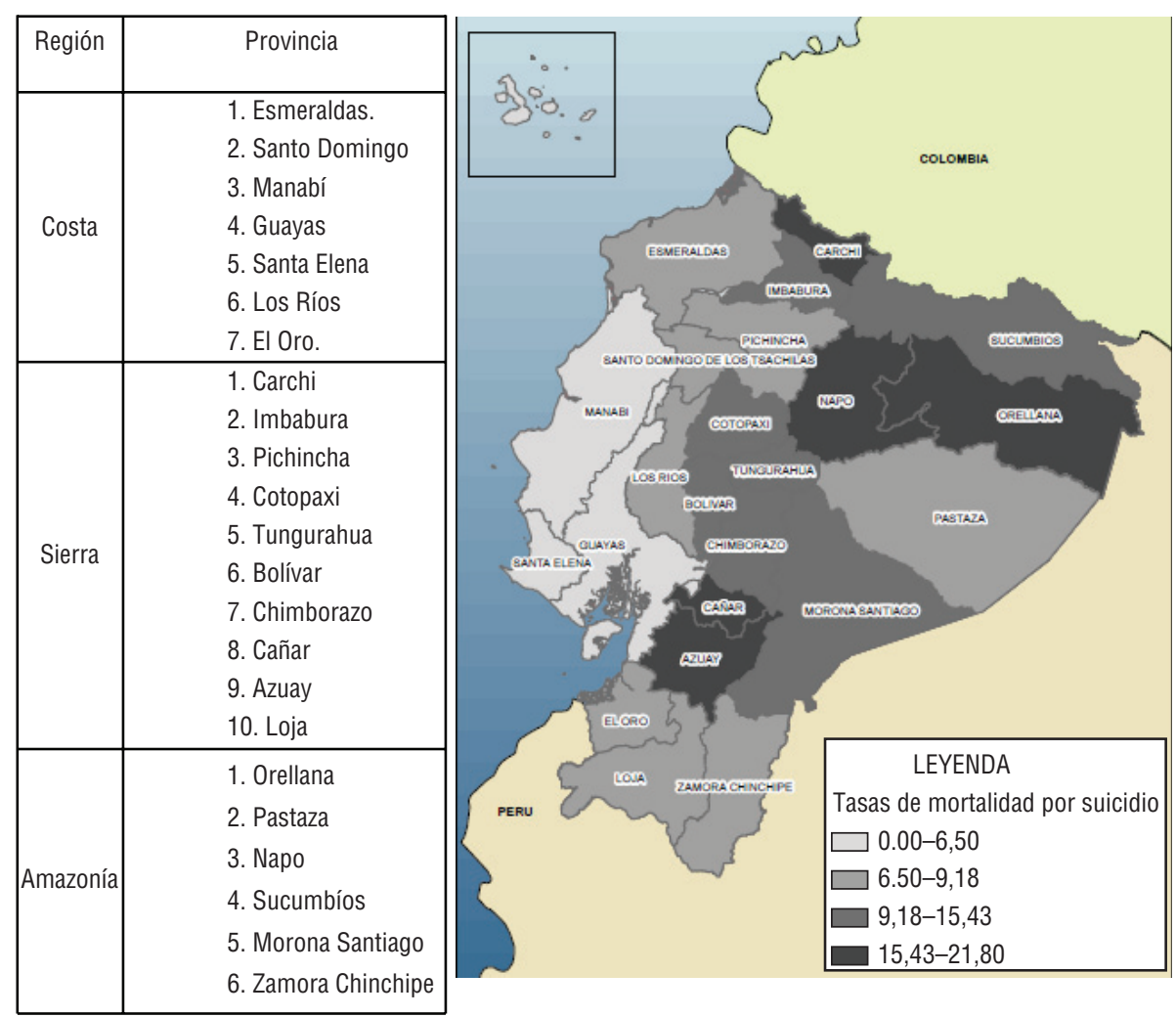

Fuente: Instituto Nacional de Estadística y Censos.

En el presente estudio, las mayores diferencias se han observado entre los 15 y los 24 años de edad. En la mayoría de los países, los hombres tienen un mayor riesgo de cometer suicidio que las mujeres y las mujeres cometen más intentos suicidas (fenómeno conocido como paradoja del suicidio) (10), lo que se explica por factores sociales, culturales y socioeconómicos inherentes de cada sociedad $(11,12)$. producir una muerte más lenta, ofrecen más oportunidades de salvar la vida (14).

Los suicidios con pesticidas son muy frecuentes y su letalidad es mayor que la de otros productos químicos y medicamentos utilizados en países occidentales (15). Más de la mitad de los suicidios de mujeres y solo una cuarta parte de los de los hombres se produjo con pesticidas, lo cual es congruente con el método usado para el suicidio en adolescentes en todo el mundo (16), con la diferencia importante de que a escala mundial solo en $7 \%$ de los suicidios de los adolescentes varones (25\% en Ecuador) y de $22 \%$ de las adolescentes (54\% en Ecuador) se utilizan pesticidas. La probabilidad de morir de las adolescentes que usan estos métodos es mayor, lo cual podría explicar por qué en Ecuador la tasa de suicidio de mujeres jóvenes es tan alta, ya que en los intentos suicidas utilizan métodos letales como los pesticidas, que reducen la probabilidad de salvarles la vida. En otros países podría haber el mismo número de intentos suicidas y menos muertes, porque los medios utilizados en muchos casos permiten salvarles la vida.

La distribución del uso de métodos para suicidarse en zonas rurales y urbanas en Ecuador es congruente con la distribución mundial. En las zonas rurales en el mundo el envenenamiento (especialmente por pesticidas) es más frecuente que en las urbanas (17), aunque el porcentaje del total de suicidios por este método no varía de manera sustancial entre ambas zonas.

Las diferencias más notables se observan por provincias, pues los suicidios que corresponden a envenenamientos variaron entre 20 y $80 \%$ de una provincia a otra. En general, las provincias con alta mortalidad por suicidio eran aquellas donde el envenenamiento por pesticidas era el método prevaleciente.

Otro factor que podría explicar la diferencia entre las tasas por regiones naturales son las etnias residentes en cada una de las provincias. Ecuador es un país pluricultural, con un $7 \%$ de población indígena, que vive sobre todo en las provincias de la Sierra y la Amazonía (7). En la Sierra, solo $15 \%$ de los suicidios de jóvenes $\mathrm{y}$ adolescentes se concentran en indígenas, mientras que en la Amazonía dicho porcentaje aumenta a $40 \%$ en esta etnia.

Las causas de las altas tasas de suicidio de indígenas jóvenes parecen estar relacionadas con factores sociales, como la 
FIGURA 2. Distribución porcentual del envenenamiento como método del suicidio en menores de 25 años por provincia, entre 2008 y 2014, Ecuador

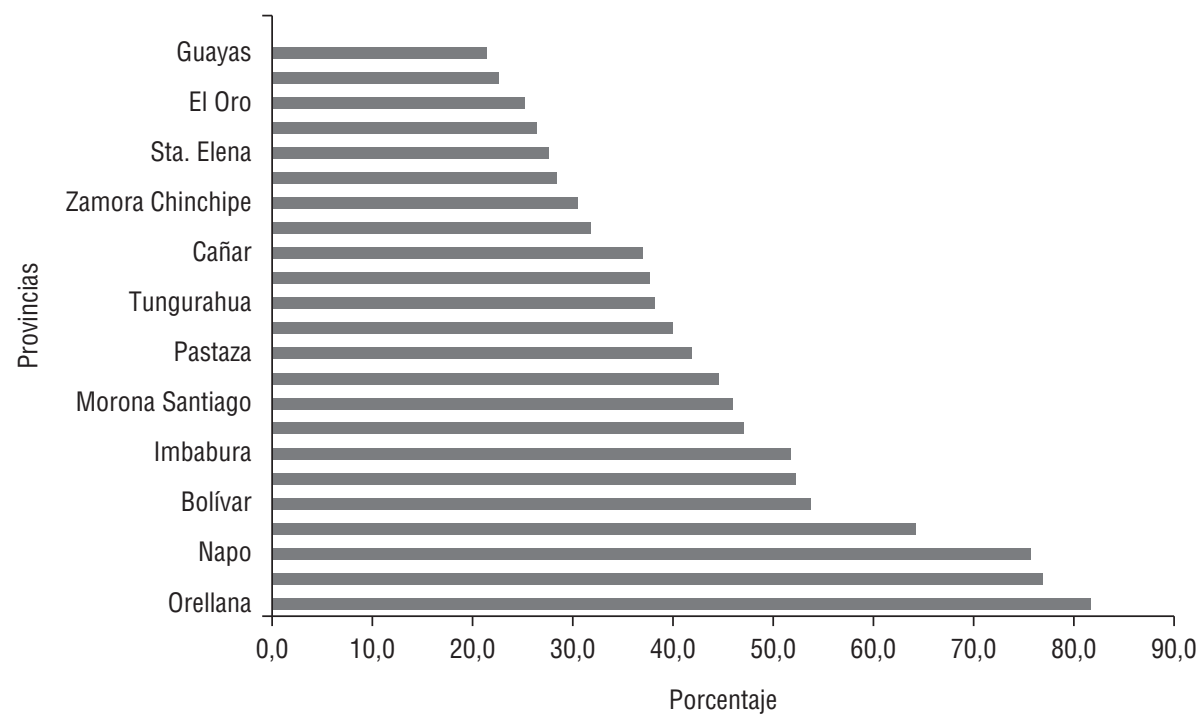

Fuente: Instituto Nacional de Estadística y Censos.

lenta enajenación de la propia cultura, el aislamiento y la aceptación del suicidio en su grupo cultural (18), así como con diversas desigualdades sociales o determinantes de la salud, como la pobreza estructural y el bajo nivel educativo. Estos grupos étnicos también están expuestos a múltiples factores de riesgo, como, por ejemplo, el alto uso de alcohol y de drogas, los problemas familiares y los de salud mental sin tratar (19).

Otro factor que puede influir en la variación de las tasas de suicidio es la desigualdad en el acceso a los servicios de salud en Ecuador. Su Ministerio de Salud Pública, a partir de la implementación del Modelo de Atención Integral en Salud (MAIS), se está esforzando en ofrecer atención médica, y también psicológica, gratuita a toda la población (20). Sin embargo, dichos servicios no están distribuidos equitativamente, lo que afecta especialmente a la población residente en zonas rurales y urbano-marginales (21). Tanto la atención médica oportuna de los intentos de suicidio como su atención psicológica o psiquiátrica adecuada son unas de las mejores herramientas para prevenir el suicidio de los jóvenes (22). La carencia de dichos servicios en ciertas zonas podría ser otro factor responsable, toda vez que aumenta la brecha entre las tasas de suicido en el país.

Otra particularidad de este país es la alta prevalencia de síntomas depresivos en los jóvenes ecuatorianos, que en dos investigaciones mostró afectar alrededor de $30 \%$ de los estudiantes de secundaria $(23,24)$. Las intervenciones diseñadas para tratarlos deben tener en cuenta este hecho como uno de los mayores factores de riesgo del suicidio (25), adaptar, además, los programas de prevención a esta realidad, así como ofrecer intervenciones personalizadas psicológicas o psiquiátricas a jóvenes en riesgo (26).

Entre las limitaciones que presentan los registros de suicidios destacan las dificultades de índole burocrática: la falta de regularización de los registros de muerte y la transición de la CIE-9 a la CIE-10, los escollos que plantea la identificación de algunos suicidios (por ejemplo, en casos de accidentes automovilísticos, sobredosis de drogas, ahogamientos, caídas), junto con las que añade el estigma social que supone para las familias (27).

Es posible que por estas causas en Ecuador exista un subregistro importante del suicidio, pues se supone que bajo la categoría de causas externas indeterminadas se esconden muchos suicidios (28). Teniendo en cuenta este subregistro, los análisis realizados en el presente estudio pueden considerarse incompletos, aunque sean de todos modos importantes.

Según las recomendaciones de varios autores $(22,29)$, la restricción del acceso a pesticidas y a otros productos químicos, los programas de prevención universales y en centros educativos desplegados en las áreas con las tasas de suicidio más altas, y las intervenciones específicas psicoterapéuticas y psiquiátricas podrían ayudar a reducir los suicidios de jóvenes en Ecuador. La restricción de medios para consumarlo es fundamental en la prevención del suicidio en jóvenes, porque la característica impulsividad relacionada con esta etapa del desarrollo psicológico les torna más propensos a comportamientos suicidas letales si el medio para consumarlo está a su alcance (30).

Estudios epidemiológicos realizados en varios países muestran que la tasa de suicidios se reduce después de haber restringido el acceso a los pesticidas más letales $(31,32)$. Siguiendo este ejemplo, se supone que la implantación de políticas dirigidas a restringir el uso de pesticidas y a concienciar a la población del peligro que entraña para los jóvenes la tenencia de pesticidas, de otros productos químicos y de medicamentos podría contribuir a salvar muchas vidas. Algunos estudios realizados en India y Sri Lanka indican que las intervenciones que ofrecen a los campesinos cajas fuertes para guardar pesticidas y otros productos químicos han ayudado a aumentar la conciencia sobre el cuidado que debe tenerse con estas sustancias e incluso a reducir el número de muertes por suicidio $(15,32)$.

Pese a que cada país tiene sus particularidades y a que las estrategias para reducir el suicidio deben adaptarse a cada cultura específica, se recomienda guiarse por las intervenciones que han funcionado en otros países. Una intervención específica dirigida al control del uso de todo tipo de productos químicos, como mantener guardados venenos y pesticidas en cajas fuertes, debe ser uno de los ejes principales en la prevención del suicidio de jóvenes y adolescentes en Ecuador. Para que estas intervenciones sean más exitosas es importante dirigirlas a las provincias donde el número de suicidios por envenenamiento es muy alto. En las provincias de Esmeraldas, Santo Domingo, Bolívar, Napo, Sucumbíos y Orellana se podrían esperar buenos resultados si los esfuerzos se encaminaran a restringir o dificultar el acceso a estos medios.

Por otra parte, se dispone de pruebas sobre la efectividad de programas escolares universales para reducir ideaciones e intentos suicidas, por lo que se recomienda implantarlos en las provincias con las tasas de suicidio más altas: Carchi, Napo, Pastaza, Azuay, Cotopaxi y Bolívar. 
Los programas de salud mental en algunas regiones de Ecuador, como la Amazonía, deben adaptarse a las peculiaridades de los pueblos indígenas residentes es ellas. Los resultados obtenidos ponen claramente de manifiesto las vulnerabilidades y las desigualdades en la distribución del suicidio y, por ello, han de tenerse presentes al diseñar intervenciones dirigidas específicamente a estos grupos.

Además, es recomendable vigilar a los adolescentes y jóvenes tras un intento suicida, pues en ellos se mantiene un riesgo alto de volver a intentarlo. Por esta razón, la OMS propone realizar un

1. Kolves K, De Leo D. Regions with the Highest Suicide Rates for Children and Adolescents - Some Observations. J Child Adolesc Behav. 2014;2(2):1000e104.

2. Quinlan-Davidson M, Sanhueza A, Espinosa I, Escamilla-Cejudo JA, Maddaleno M. Suicide among young people in the Americas. J Adolesc Health. 2014;54(3):262-8.

3. Instituto Nacional de Estadísticas y Censos. Anuario de Estadísticas Vitales: Nacimientos y Defunciones. 2001-2014. Quito: INEC; 2015.

4. Organización Panamericana de la Salud Pública. Mortalidad por suicidio en las Américas: Informe regional. Washington, DC: OPS; 2014.

5. Ortiz-Prado E, Simbaña K, Gómez L, Henriquez-Trujillo AR, Cornejo-Leon F, Vasconez E, et al. The disease burden of suicide in Ecuador, a 15 years' geodemographic cross-sectional study (2001-2015). BMC Psychiatry. 2017;17(1):342-53.

6. McLoughlin AB, Gould MS, Malone KM. Global Trends in Teenage Suicide: 2003-2014. QJM. 2015;101(10):765-80.

7. Azuero AJ, Arreaza-Kaufman D, Coriat J, Tassinari S, Faria A, Castañeda-Cardona C, et al. Suicide in the Indigenous Population of Latin America: A Systematic Review. Rev Colomb Psiquiatr. 2017;46(4):237-42.

8. Hee Ahn M, Park S, Ha K, Choi SH, Hong JP. Gender ratio comparisons of the suicide rates and methods in Korea, Japan, Australia, and the United States. J Affect Disord. 2012;142(1):161-5.

9. Wasserman D, Cheng Q, Jiang G. Global suicide rates among young people aged 15-19. World Psychiatry. 2005;4(2):114-20.

10. Canetto SS, Sakinofsky I. The Gender Paradox in Suicide. Suicide Life-Threatening Behav. 1998;28(1):1-23.

11. Payne S, Swami V, Stanistreet DL. The social construction of gender and its influence on suicide: a review of the literature. J Mens Health. 2008;5(1):23-35.

12. McKay K, Milner A, Maple M. Women and suicide: beyond the gender paradox. Int J Cult Ment Health. 2014;7(2):168-78. seguimiento periódico, por lo menos de un año, mediante visitas, llamadas, cartas $\mathrm{u}$ otras formas de comunicación con los afectados que sean sostenibles para el sistema de salud del país (2).

Las características epidemiológicas consideradas en este estudio pueden ofrecer pautas útiles para el análisis y la prevención adecuados de los suicidios en Ecuador y en otros países de la Región que compartan con este país características culturales, étnicas, geográficas y socioeconómicas.

Agradecimientos. Los autores agradecen a las autoridades de la Pontificia

\section{REFERENCIAS}

13. Beautrais AL. Gender issues in youth suicidal behaviour. Emerg Med. 2002;14(1): 35-42.

14. Mergl R, Koburger N, Heinrichs K, Székely A, Tóth MD, Coyne J, et al. What Are Reasons for the Large Gender Differences in the Lethality of Suicidal Acts? An Epidemiological Analysis in Four European Countries. PLoSOne.2015;6;10(7):e0129062. Disponible en: https://doi.org/10.1371/ journal.pone.0129062 Acceso el 30 de mayo de 2018.

15. Mishara BL. Prevention of Deaths from Intentional Pesticide Poisoning. Crisis. 2007;28(Suppl 1):10-20.

16. Kõlves K, de Leo D. Suicide methods in children and adolescents. Eur Child Adolesc Psychiatry. 2017;26(2):155-64.

17. Hirsch JK, Cukrowicz KC. Suicide in rural areas: An updated review of the literature. J Rural Ment Health. 2014;38(2):65-78.

18. Niezen R. The Durkheim-Tarde debate and the social study of aboriginal youth suicide. Transcult Psychiatry. 2014;52(1):96-114.

19. McKenzie K, Serfaty M, Crawford M. Suicide in ethnic minority groups. $\mathrm{Br} \mathrm{J}$ Psychiatry. 2003;183(2):100-1.

20. Ministerio de Salud Pública del Ecuador. Manual del Modelo de Atención Integral de Salud Familiar, Comunitario e Intercultural. Quito; MSP: 2013.

21. López-Cevallos D, Chi C, Ortega F. Consideraciones para la transformación del sistema de salud del Ecuador desde una perspectiva de equidad .Rev Salud Publica. 2014;16(3):347-59.

22. Zalsman G, Hawton K, Wasserman D, van Heeringen K, Arensman E, Sarchiapone $\mathrm{M}$, et al. Suicide prevention strategies revisited: 10-year systematic review. Lancet Psychiatry. 2016;3(7):646-59.

23. Schnell T, Gerstner R, Krampe H. Crisis of meaning predicts suicidality in youth independently of depression. Crisis. 2018;Feb 23:1-10. doi: 10.1027/0227-5910/a000503. Disponible en: https://doi.org/10.1027/ 0227-5910/a000503 Acceso el 24 de febrero de 2018 .
Universidad Católica, Sede Santo Domingo, el haber impulsado esta investigación en beneficio de los adolescentes de Ecuador.

Financiación. El presente estudio no ha recibido financiación externa.

Conflictos de interés. Los autores declaran no tener conflictos de interés.

Declaración. Las opiniones expresadas en este manuscrito son responsabilidad de los autores y no reflejan necesariamente los criterios ni la política de la RPSP/ PAJPH y/o de la OPS.
24. Gerstner R. Factores predictores y protectores para tendencias suicidas en adolescentes en una zona afectada por el terremoto del 16 de abril en Ecuador. [Tesis.] Quito: Universidad San Francisco de Quito; 2017. Disponible en: http:/ / repositorio.usfq.edu. ec/handle/23000/21 Acceso el 30 de mayo de 2018.

25. Chesney E, Goodwin GM, Fazel S. Risks of all-cause and suicide mortality in mental disorders: a meta-review. World Psychiatry. 2014;13(2):153-60.

26. Gould MS, Greenberg T, Velting D, Shaffer D. Youth Suicide Risk and Preventive Interventions: A Review of the Past 10 Years. J Am Acad Child Adolesc Psychiatry. 2003;42(4):386-405.

27. De Leo D. Can We Rely on Suicide Mortality Data? Crisis. 2015;36(1):1-3.

28. González-Andrade F, López-Pulles R, Gascón S, García Campayo J. Epidemiological issues regarding suicides in Ecuador: an 8-year report. J Public Health. 2010; 19(2):161-9.

29. Sinyor M, Tse R, Pirkis J. Global trends in suicide epidemiology. Curr Opin Psychiatr. 2017;30(1):1-6.

30. Hawton K, Saunders KEA, O'Connor RC Self-harm and suicide in adolescents. Lancet. 2012;379:2373-82.

31. Vijayakumar L, Satheesh-Babu R. Does 'No Pesticide' Reduce Suicides? Int J Soc Psych. 2009;55(5):401-6.

32. Vijayakumar L, Jeyaseelan L, Kumar S, Mohanraj R, Devika S, Manikandan S. A central storage facility to reduce pesticide suicides - a feasibility study from India. BMC Public Health. 2013;13(1):850.

Manuscrito recibido el 18 de diciembre de 2017. Aceptado para publicación, tras revisión, el 5 de abril de 2018. 
ABSTRACT Objective. To collect information - and provide it to decision-makers in health programs in general and mental health programs in particular - on epidemiological patterns in suicide deaths among young adults and adolescents in Ecuador.

\section{Epidemiology of suicide among adolescents and young adults in Ecuador}

Keywords Suicide; adolescent; epidemiology; Ecuador.
Methods. A cross-sectional ecological study was conducted using data obtained from records of deaths by suicide among young adults and adolescents in Ecuador from 2001 to 2014. For analysis, results were disaggregated by geographic region, sex, age, ethnic group and suicide method. Rates of death by suicide (per 100,000 population) and relative risks (RR) for suicide were estimated by sex and region.

Results. Between 2001 and 2014, 4,855 suicides were recorded among adolescents and young adults. The highest estimated risk was found among males aged 15 to 24 years and adolescents living in the Amazon region, followed by those living in the Andean region. Mestizos were the ethnic group with the greatest number of suicides, although $40 \%$ of young people who commit suicide in the Amazon region are indigenous. The most frequent method was by hanging, followed by pesticide poisoning.

Conclusions. Suicide among adolescents and young adults is an important public health problem in Ecuador. Since various psychological, social, and cultural influences come into play, there is wide variation among regions, age groups, and ethnic groups. Restricting access to pesticides and other chemical products, implementing universal prevention programs and programs in education centers in the areas with the highest rates, and targeting vulnerable populations for specific interventions could help reduce the suicide rate among young people in Ecuador.
RESUMO

\section{Epidemiologia do suicídio em adolescentes e jovens no Equador}

\author{
Palavras-chave
}

Objetivo. Coletar e prover informação aos responsáveis pela tomada de decisão nos programas de saúde, em geral, e de saúde mental, em particular, quanto às características epidemiológicas do suicídio em jovens e adolescentes equatorianos.

Métodos. Um estudo ecológico transversal foi realizado com dados dos registros de mortalidade por suicídio em adolescentes e jovens do Equador entre 2001 e 2014. A análise foi desagregada por região de origem, sexo, idade, grupo étnico e método usado para o suicídio. Foram estimados os índices de mortalidade por suicídio (por 100.000 habitantes) e o risco relativo (RR) de suicídio por sexo e região.

Resultados. No período entre 2001 e 2014, foram registrados 4.855 suicídios em adolescentes e jovens. Maior risco de suicídio foi estimado em indivíduos do sexo masculino com idade de 15 a 24 anos e adolescentes residentes na Amazônia e região de La Sierra. O grupo étnico com maior número de casos de suicídio foi o dos mestiços, embora 40\% dos casos de suicídio em jovens na Amazônia ocorreram em indígenas. Os métodos mais usados foram enforcamento e, em segundo lugar, envenenamento por pesticidas.

Conclusões. O suicídio de adolescentes e jovens é um importante problema de saúde pública no Equador. Como se trata de um fenômeno determinado por uma conjunção de fatores psicológicos, sociais e culturais, observa-se uma grande variação segundo região, faixa etária e grupo étnico. Restrição ao acesso a pesticidas e a outros produtos químicos, programas de prevenção universais e em centros educacionais em áreas com as taxas mais elevadas e intervenções específicas voltadas aos grupos vulneráveis podem contribuir para reduzir os casos de suicídio entre jovens no Equador.

Suicídio; adolescente; epidemiologia; Equador. 